G1-442a.

8) Steele D, et al. 48th ICAAC/IDSA 46th annual meeting, Washington DC, October 25-28, 2008; G1-442b.

9) Hanquet G, et al. 27th European Society for Paediatric Infectious Diseases (ESPID) Brussels, Belgium, 9-13 June 2009; abstract O25.

10) Paulke-Korinek $M$, et al. Pediatr Infect Dis J 2010; 29: 319-23.

11) CDC. MMWR Morb Mortal Wkly Rep 2008; 57 : 697-700.

12) Aladin F, et al. J Clin Virol 2010; 47: 340-4.

13) Iturriza-Gomara $M$, et al. J Infect Dis 2009; 200 Suppl 1: S215-21.

14) World Health Organization. Wkly Epidemiol Rec 2009; 84: 220-36.

7

\section{OPTIMISING PREVENTION OF PNEUMOCOCCAL DISEASE WITH PNEUMOCOCCAL CONJUGATE VACCINES PFIZER SYMPOSIUM, 23 OCTOBER 2010, 13:00-14:00}

\section{N. Stephens}

ESAT Vaccines, Pfizer, Paris, France

Paediatricians play a critical role in the battle against pneumococcal diseases, with a front-line position in its ongoing surveillance and prevention. The addition of pneumococcal conjugate vaccines (PCVs) to the armamentarium has dramatically changed the landscape in this battle; PCV7 was introduced almost a decade ago, and has been shown to have had a strong impact on all pneumococcal diseases caused by vaccine serotypes.

This symposium will open by looking at the burden of invasive and non-invasive pneumococcal disease across Europe, comparing the incidence and prevalence pre- and post-PCV7, and examining the impact of the different vaccination schedules in force.

Putting all this into practice, a member of our distinguished faculty will present an interactive case study on paediatric empyema, giving the audience the chance to vote and share their views on treatment strategies for invasive pneumococcal diseases.

Finally, we will turn to consider the remaining burden of pneumococcal disease, looking at the broader coverage of the 13-valent PCV formulation, and the practicalities of transitioning from PCV7, as well as the opportunities for providing broader protection to already fully vaccinated children.

We very much hope you will join us for what promises to be a lively and educational event as we consider how best to optimise the prevention of pneumococcal diseases with PCVs.

\section{8}

\section{TREATMENT OPTIONS FOR NP-C: CHANGING OUR APPROACH TO THE DISEASE}

M. Patterson

\section{Division of Child and Adolescent Neurology, Mayo Clinic, Rochester, MN, USA}

Definitive disease-modifying therapy for NiemannPick disease, type C (NPC) would either correct the primary genetic defect or replace the deficient gene product [1]. Neither strategy is currently applicable to patients with NPC1 mutations, but replacement of the NPC2 protein by haematopoietic stem cell transplantation is theoretically possible, although proof of principle in humans is lacking.

Alternative approaches involve interdicting pathways downstream to the primary mutation that lead to cellular dysfunction. These approaches include reduction of substrate burden, replacement of deficient downstream products, suppression of inflammation, inhibition of apoptosis and activation of alternate pathways. Of these approaches, only substrate reduction therapy has been studied and approved for use in humans.

Miglustat is an iminosugar that competitively inhibits glucosylceramide synthase, the enzyme catalysing the rate-limiting step in the synthesis of higher order glycosphingolipids. Miglustat has been shown to delay the onset of symptoms in the NPC murine model. Both prospective and retrospective human studies have also found evidence of a beneficial effect on disease progression in NPC [2-5]. The availability of such a treatment mandates early diagnosis of patients with NPC, since those without irreversible disease will be likely to benefit most. Currently, there is no readily available test for NP. $\mathrm{C}$, but in future, oxysterols or other biomarkers may facilitate screening for NPC. 
Guidelines have been published for the general medical management of people with NPC [6]. It is essential that physicians take a comprehensive approach to managing NPC, since patients are most likely to benefit when disease-modifying therapy is combined with meticulous general medical care and symptom management.

\section{References:}

1. Patterson and Platt. Biochim Biophys Acta 2004;1685(1-3):77-82

2. Patterson. et al. J Child Neurol 2010;25(3):300305

3. Patterson. et al. Lancet Neurol 2007;6(9):765772

4. Pineda et al. Mol Genet Metab 2009;98(3):243249

5. Wraith et al. Mol Genet Metab 2010;99(4):351357

6. Wraith et al. Mol Genet Metab 2009;98(1-2):152165

9

\section{IDENTIFYING NP-C EARLY IN PAEDIATRIC PATIENTS}

\section{F.A. Wijburg}

Department of Paediatrics, Amsterdam Medical Centre, Amsterdam, The Netherlands

Niemann-Pick disease, type C (NP-C) is an autosomal recessive, progressive and ultimately fatal, neurovisceral lysosomal storage disorder involving the accumulation of unesterified cholesterol and glycolipids. Two genes (NPC1 and NPC2) are involved in its pathogenesis. The estimated incidence of NP-C is 1:150,000 live births; however, the true incidence is probably significantly higher, as there is ample evidence that many patients remain undiagnosed.

Clinical presentation in NP-C is extremely heterogenous, often resulting in misdiagnosis. The first signs and symptoms may occur from the prenatal period; through to childhood and late adulthood. The absence of a simple diagnostic test for NP-C precludes the inclusion of the disease in general metabolic screening panels.

The following signs and symptoms should lead to inclusion of NP-C in a differential diagnosis in paediatric patients: prolonged unexplained neonatal jaundice; hepatosplenomegaly or isolated splenomegaly; and neurological signs including vertical supranuclear gaze palsy, ataxia and/or dystonia, seizures and/or cataplexia, and early and progressive dementia.

At present, definitive diagnosis depends on demonstrating impaired cholesterol transport and homeostasis in cultured skin fibroblasts followed by genetic testing for mutations in NPC1 and NPC2. A simple diagnostic tool based on a scoring system for various visceral, neurological and psychiatric signs and symptoms is currently being developed to facilitate decision-making regarding the need for diagnostic biochemical or genetic studies for NP. C.

With the recent approval of miglustat (Zavesca ${ }^{\circledR}$ ) as disease-modifying therapy for NP-C, treatment can now be aimed toward stabilising neurological disease. Early diagnosis of NPC has, thus, become even more important.

10

\section{DIFFERENTIAL DIAGNOSIS IN PATIENTS WITH DEVELOPMENTAL DELAY: FOCUS ON INBORN ERRORS OF METABOLISM}

\section{P. Gissen}

\section{University of Birmingham, Birmingham, UK}

Developmental delay (DD) is common in paediatric practice and is present in $5-10 \%$ of children. Detailed evaluation can yield a definitive diagnosis in more than half of these cases and a substantial proportion of these are caused by chromosomal abnormalities and defects in the genes important for embryo development.

An inborn error of metabolism (IEM) can be identified in approximately $1 \%$ of patients with DD, but although a diagnosis of IEM is rare, accurate identification could make a big difference to the prognosis for the affected child, as well as counselling of the family and management of siblings. A diagnosis of IEM is more likely in the presence of additional abnormalities and developmental regression. The incidence of IEMs and the frequency of individual disorders vary depending on ethnicity.

IEMs can present at any age, but earlier diagnosis increases the chance of an improved prognosis, particularly in cases where treatments are available. 